\title{
Phosphorus Distribution between Soda- and Lime-based \\ Fluxes and Carbon-saturated Iron Melts*
}

\section{By Ryo INOUE** and Hideaki SUITO**}

\begin{abstract}
Synopsis
The phosphorus distribution ratios between carbon-saturated iron melts and $\mathrm{Na}_{2} \mathrm{O}-\mathrm{SiO}_{2}, \mathrm{Na}_{2} \mathrm{O}-\mathrm{SiO}_{2}-\mathrm{MO}(\mathrm{M}=\mathrm{Mg}, \mathrm{Ca} \mathrm{Ba}), \mathrm{CaF}_{2}-\mathrm{CaO}$ or $\mathrm{CaF}_{2}-\mathrm{CaO}-\mathrm{Al}_{2} \mathrm{O}_{3}$ slags have been obtained as a function of the slag compositions and the temperatures. The distribution ratios in the $\mathrm{Na}_{2} \mathrm{O}$ $\mathrm{SiO}_{2}-\mathrm{MO}(\mathrm{M}=\mathrm{Mg}, \mathrm{Ca}, \mathrm{Ba})$ system were found to decrease when $\mathrm{Na}_{2} \mathrm{O}$ was replaced by $\mathrm{MgO}, \mathrm{CaO}$, and $\mathrm{BaO}$ at a constant $\mathrm{SiO}_{2}$ content. The temperature dependence of the phosphorus distribution ratios in the $\mathrm{CaF}_{2}-\mathrm{CaO}$ and $\mathrm{CaF}_{2}-\mathrm{CaO}-\mathrm{Al}_{2} \mathrm{O}_{3}$ systems has been observed in the temperature range between $1430^{\circ} \mathrm{C}$ and $1550^{\circ} \mathrm{C}$, while in the $\mathrm{Na}_{2} \mathrm{O}$ $\mathrm{SiO}_{2}$ system, it has not been observed at 1250 and $1350^{\circ} \mathrm{C}$. The phosphorus distribution ratios in the $\mathrm{MF}_{2}(\mathrm{MF})-\mathrm{MO}\left(\mathrm{M}_{2} \mathrm{O}\right) \quad(\mathrm{M}=$ $\mathrm{Mg}, \mathrm{Ca}, \mathrm{Ba}, \mathrm{Na}$ ) system were found to decrease in the order of $\mathrm{Na}>\mathrm{Ba}>\mathrm{Ca}>\mathrm{Mg}$.
\end{abstract}

\section{Introduction}

In recent years many laboratory and plant data on the dephosphorization of hot metal by using sodaand lime-based fluxes have been reported in our country. Very little work, however, is currently available in the published literatures on the thermodynamic data for the activity of phosphorus oxide in these fluxes. The reason for this is mainly due to the experimental difficulties in the temperature range of a hot metal pretreatment. Iwasaki et al. ${ }^{1)}$ have obtained the phosphorus distribution ratios between lime-based fluxes and solid iron at a controlling oxygen potential in the temperature range of a hot metal pretreatment. In their experimental method, however, the ratios between the solid iron and the fluxes containing the strong base such as a sodium oxide become too high with the decreasing temperature. The oxygen potentials therefore must be lowered to such an extent that the phosphorus content in metal can be determined within the accuracy of chemical analysis. The phosphorus partitions ${ }^{2-4)}$ between liquid iron and slags obtained in the temperature between $1550{ }^{\circ} \mathrm{C}$ and $1650^{\circ} \mathrm{G}$ have been extrapolated to the temperatures in a hot metal pretreatment in order to discuss the dephosphorization of hot metal. ${ }^{\text {) }}$

The present authors have previously obtained the sulfur distribution ratios between the $\mathrm{Na}_{2} \mathrm{O}-\mathrm{SiO}_{2}$ slags and carbon-saturated iron melts at 1250 and $\left.1350{ }^{\circ} \mathrm{C}^{6}{ }^{6}\right) \quad$ These results indicate that the slag compositions can be chosen up to the $\mathrm{Na}_{2} \mathrm{O} / \mathrm{SiO}_{2}=1.5$ (molar ratio) in the above temperature range without the significant change in the slag compositions due to the sodium evaporation, since the time for the attainment of the equilibrium can be considerably shorten by stirring a melt. The sulfur distribution ratios ${ }^{7)}$ between the $\mathrm{CaF}_{2}-\mathrm{CaO}-\mathrm{Al}_{2} \mathrm{O}_{3}$ slags and carbon-saturated iron melts under the $P_{\mathrm{CO}}=1$ atm were found to be in an excellent agreement with those calculated from the sulfide capacity, $C_{\mathrm{S}}(=(\% \mathrm{~S})$. $\left.a_{\mathrm{O}} / a_{\mathrm{S}}\right)$ obtained by the gas-slag equilibration. ${ }^{8)}$ From these experimental findings, the distribution ratios between soda- and lime-based fluxes and carbonsaturated iron melts have been obtained under the CO atmosphere. These results are compared with those obtained by other investigators by using the concept of phosphate capacity, $C_{\mathrm{P}}\left(=(\% \mathrm{P}) /\left[a_{\underline{\mathrm{P}}} \cdot a_{\underline{\mathrm{o}}}^{5 / 2}\right]\right)$.

\section{Experimental}

The details of the experimental apparatus and method are described in the previous paper ${ }^{6)}$ on the sulfur distribution. Slag (8g) and metal (30 g) were melted in a graphite crucible under a $\mathrm{CO}$ gas flow, being stirred by an alumina or graphite rod. In some of the experiments using the $\mathrm{Na}_{2} \mathrm{O}-\mathrm{SiO}_{2}$ slags, copper was used instead of iron as a metal sample. The holding time was from $6 \mathrm{~min}$ to $4 \mathrm{~h}$, depending on the slag compositions and the temperatures. The experimental temperatures for soda-based fluxes was 1250 and $1350{ }^{\circ} \mathrm{C}$. Those for $\mathrm{CaF}_{2}$-based and $\mathrm{BaF}_{2}\left(\mathrm{MgF}_{2}, \mathrm{NaF}\right)$-based fluxes were 1500 and $1350{ }^{\circ} \mathrm{C}$, respectively. The temperature dependence of phosphorus distribution ratio, $L_{\mathrm{P}}$ was studied by use of the $\mathrm{CaF}_{2}-\mathrm{CaO}$ and $\mathrm{CaF}_{2}-$ $\mathrm{CaO}-\mathrm{Al}_{2} \mathrm{O}_{3}$ fluxes. Equilibrium was approached mostly from metal side, i.e., by adding an $\mathrm{Fe}-4.5 \% \mathrm{C}-$ $1 \% \mathrm{P}$ alloy in the metal phase, but in some of the experiments for $\mathrm{Na}_{2} \mathrm{O}-\mathrm{SiO}_{2}$ and fluoride-based fluxes, it was approached from slag side also, i.e., by adding $\mathrm{NH}_{4} \mathrm{H}_{2} \mathrm{PO}_{4}, \quad 3 \mathrm{Na}_{2} \mathrm{O} \cdot \mathrm{P}_{2} \mathrm{O}_{5}, \quad 3 \mathrm{CaO} \cdot \mathrm{P}_{2} \mathrm{O}_{5}, \quad 2 \mathrm{BaO} \cdot \mathrm{P}_{2} \mathrm{O}_{5}$ and $3 \mathrm{MgO} \cdot \mathrm{P}_{2} \mathrm{O}_{5}$. In the experiment using copper, the phosphorus equilibrium was approached both from metal side (initial content of $0.1 \% \mathrm{P}$ ) and from slag side (initial content of $2 \% \mathrm{P}_{2} \mathrm{O}_{5}$ ). Materials used in this work were the same as those described elsewhere ${ }^{6)}$ except for $\mathrm{NaF}(99.0 \%), \mathrm{CaF}_{2}$ and $\mathrm{BaF}_{2}$ (Merck Suprapur) and $\mathrm{MgF}_{2}(99.9 \%$ ). The method for analysis of the slag and metal compositions were the same as those described in detail elsewhere ${ }^{2,6)}$ except for fluorine, barium, total iron and phosphorus in the slags.

The fluorine content in the slag was analyzed by the ionic electrode method and/or the La-alizarin complexone absorptiometry (JIS-K-0101) after the

* Partly presented to the 105th ISIJ Meeting, April 1983, A25, at The University of Tokyo in Tokyo. Manuscript received June 20, 1984; accepted in the final form on August 16, 1984. (C) 1985 ISIJ

** Research Institute of Mineral Dressing and Metallurgy, Tohoku University, Katahira, Sendai 980. 
alkali fusion of samples with $\mathrm{SiO}_{2} \cdot{ }^{9,10)}$ The content of $\mathrm{Ba}$ was determined by the $\mathrm{BaSO}_{4}$ gravimetric method. ${ }^{10)}$ Since the analytical results for phosphorus and total iron in the slags were significantly influenced by the residual metallic iron in the slags after magnetic separation, the following methods were used. As to the samples with $\mathrm{NaF}$ or soda-based slags, the bromine methanol was used to remove the metallic iron in the residue after filtration by hot water, and phosphorus and total iron were determined by molybdenum blue absorptiometry (JIS-M-8216) and 1, 10-Phenanthroline absorptiometry (JIS-K-1200), respectively. After the removal of the metallic iron in the slags containing $\mathrm{MgF}_{2}, \mathrm{CaF}_{2}$ and $\mathrm{BaF}_{2}$ by bromine methanol, the alkali fusion of the residue with $\mathrm{SiO}_{2}$ was made. Then, total iron was determined by $1,10-$ Phenanthroline absorptiometry (JIS-M-8850, R-2522, R-3101) or 2, 2'-Dipyridyl absorptiometry (JIS-K1417). As to phosphorus, $\mathrm{pH}$ was controlled after the addition of $\mathrm{FeCl}_{3}$ to the solution so as to obtain the precipitate. ${ }^{11}$. After dissolution of this precipitate, phosphorus was determined by molybdenum blue absorptiometry.

\section{Results}

The $\mathrm{Na}_{2} \mathrm{O}-\mathrm{SiO}_{2}-\mathrm{P}_{2} \mathrm{O}_{5}(\leq 19 \%), \mathrm{Na}_{2} \mathrm{O}-\mathrm{SiO}_{2}-\mathrm{MO}$ $(\mathrm{M}=\mathrm{Mg}, \mathrm{Ca}, \mathrm{Ba})-\mathrm{P}_{2} \mathrm{O}_{5}(\leq 4 \%)$ and $\mathrm{Na}_{2} \mathrm{O}-\mathrm{SiO}_{2}-$
$\mathrm{CaF}_{2}(\leq 19 \%)-\mathrm{P}_{2} \mathrm{O}_{5}(<2 \%)$ systems are used as the soda-based fluxes, whereas the $\mathrm{CaF}_{2}-\mathrm{CaO}-\mathrm{P}_{2} \mathrm{O}_{5}$ $(<70 \mathrm{ppm}), \mathrm{BaF}_{2}-\mathrm{BaO}-\mathrm{P}_{2} \mathrm{O}_{5}(\leq 2 \%), \mathrm{MgF}_{2}-\mathrm{MgO}-$ $\mathrm{P}_{2} \mathrm{O}_{5}(<5 \mathrm{ppm}), \mathrm{NaF}-\mathrm{Na}_{2} \mathrm{O}-\mathrm{P}_{2} \mathrm{O}_{5}(<0.02 \%), \mathrm{CaF}_{2}-$ $\mathrm{BaO}-\mathrm{P}_{2} \mathrm{O}_{5}(<20 \mathrm{ppm}), \mathrm{BaF}_{2}-\mathrm{CaO}-\mathrm{P}_{2} \mathrm{O}_{5}(<0.02 \%)$, $\mathrm{CaF}_{2}-\mathrm{MgO}-\mathrm{P}_{2} \mathrm{O}_{5}(<20 \mathrm{ppm})$ and $\mathrm{CaF}_{2}-\mathrm{CaO}-\mathrm{Al}_{2} \mathrm{O}_{3}-$ $\mathrm{P}_{2} \mathrm{O}_{5}$ (<70 ppm) systems are used as the fluoridebased fluxes. The chemical compositions of the slags and the metals ( $\mathrm{P}, \mathrm{Si}, \mathrm{S})$ before and after the experiments in the soda-based fluxes are listed in Tables 1 to 3 and those after the experiments in the fluoridebased fluxes are summarized in Tables 4 and 5 .

Figure 1 shows the results for $L_{\mathrm{P}}(=(\% \mathrm{P}) /[\% \mathrm{P}])$ which was obtained in the $\mathrm{Na}_{2} \mathrm{O}-\mathrm{SiO}_{2}$ system equilibrated with carbon-saturated iron melts at 1250 and $1350^{\circ} \mathrm{C}$. The values for $L_{\mathrm{P}}$ in the $\mathrm{Na}_{2} \mathrm{O} \cdot \mathrm{SiO}_{2}$ $\mathrm{MO} \cdot \mathrm{SiO}_{2}, 3 \mathrm{Na}_{2} \mathrm{O} \cdot 2 \mathrm{SiO}_{2}-3 \mathrm{MO} \cdot 2 \mathrm{SiO}_{2}(\mathrm{M}=\mathrm{Mg}, \mathrm{Ca}$, $\mathrm{Ba})$ and $3 \mathrm{Na}_{2} \mathrm{O} \cdot 2 \mathrm{SiO}_{2}-\mathrm{CaO}\left(\mathrm{CaF}_{2}\right)$ pseudo-binary systems at $1250{ }^{\circ} \mathrm{C}$ are shown in Fig. 2. Figure 3 indicates the values for $L_{\mathrm{P}}$ in the $\mathrm{NaF}-\mathrm{Na}_{2} \mathrm{O}\left(1350^{\circ} \mathrm{C}\right)$, $\mathrm{CaF}_{2}-\mathrm{CaO}\left(1500^{\circ} \mathrm{C}\right), \mathrm{BaF}_{2}-\mathrm{BaO}\left(1350^{\circ} \mathrm{C}\right)$ and $\mathrm{MgF}_{2}-\mathrm{MgO}\left(1350{ }^{\circ} \mathrm{C}, 1500^{\circ} \mathrm{C}\right.$ ) systems. The results for the reciprocal salt mixture: $\mathrm{BaF}_{2}-\mathrm{CaO}\left(1350^{\circ} \mathrm{C}\right)$, $\mathrm{CaF}_{2}-\mathrm{BaO}\left(1500^{\circ} \mathrm{C}\right)$ and $\mathrm{CaF}_{2}-\mathrm{MgO}\left(1500^{\circ} \mathrm{C}\right)$ systems are shown in Fig. 4. The values for $L_{\mathrm{P}}$ in the $\mathrm{CaF}_{2}-\mathrm{CaO}-\mathrm{Al}_{2} \mathrm{O}_{3}$ system $\left(1430{ }^{\circ} \mathrm{C} \sim 1550{ }^{\circ} \mathrm{C}\right)$ are shown in Fig. 5.

Table 1. Experimental data for soda-based flux. (wt $\%$ )

\begin{tabular}{|c|c|c|c|c|c|c|c|c|c|c|c|c|c|}
\hline \multirow{2}{*}{$\begin{array}{c}\text { Temp. } \\
\left({ }^{\circ} \mathrm{C}\right)\end{array}$} & \multirow{2}{*}{ No. } & \multicolumn{4}{|c|}{ Initial } & \multicolumn{7}{|c|}{ Final } & \multirow{2}{*}{$\begin{array}{l}\text { Time } \\
(\mathrm{min})\end{array}$} \\
\hline & & $\underline{P}$ & $\left(\mathrm{P}_{2} \mathrm{O}_{5}\right)$ & $\left(\mathrm{Na}_{2} \mathrm{O}\right)$ & $\left(\mathrm{SiO}_{2}\right)$ & $\underline{P}$ & $\underline{\mathrm{Si}}$ & $\left(\mathrm{P}_{2} \mathrm{O}_{5}\right)$ & $\left(\mathrm{Na}_{2} \mathrm{O}\right)$ & $\left(\mathrm{SiO}_{2}\right)$ & $\left(\mathrm{Fe}_{t} \mathrm{O}\right)$ & $\left(\mathrm{Al}_{2} \mathrm{O}_{3}\right)$ & \\
\hline \multirow{15}{*}{1250} & 91 & 1.10 & - & 26.1 & 73.89 & 1.04 & 0.086 & 0.0100 & 25.2 & 73.64 & 0.843 & 0.3 & 122 \\
\hline & 92 & 1.10 & - & 41.2 & 58.79 & 0.964 & 0.022 & 0.0113 & 36.1 & 62.34 & 0.462 & 1.1 & 119 \\
\hline & 93 & 1.10 & - & 50.8 & 49.23 & 0.916 & 0.005 & 0.435 & 47.9 & 50.51 & 0.410 & 0.7 & 38 \\
\hline & 94 & 1.10 & - & 60.5 & 39.53 & 0.635 & 0.004 & 3.11 & 55.4 & 40.51 & 0.133 & 0.8 & 14 \\
\hline & 95 & 0.005 & 1.90 & 27.4 & 70.66 & 0.189 & 0.071 & 0.111 & 27.6 & 70.86 & 0.895 & 0.5 & 117 \\
\hline & 96 & 0.005 & 1.90 & 51.0 & 47.07 & 0.110 & $\operatorname{tr}$. & 0.857 & 47.0 & 50.55 & 0.580 & 1.0 & 46 \\
\hline & 97 & 0.005 & 1.79 & 60.5 & 37.74 & 0.0070 & 0.004 & 1.92 & 56.3 & 39.98 & 0.409 & 1.3 & 17 \\
\hline & 130 & 1.10 & - & 24.2 & 75.78 & 1.01 & 0.175 & 0.0086 & 24.0 & 74.77 & 0.765 & 0.4 & 232 \\
\hline & 131 & 1.10 & - & 41.2 & 58.79 & 0.958 & 0.040 & 0.0079 & 36.2 & 61.73 & 0.437 & 1.6 & 131 \\
\hline & 132 & 0.552 & 0.48 & 50.3 & 49.24 & 0.467 & tr. & 0.559 & 48.2 & 50.01 & 0.418 & 0.8 & 28 \\
\hline & 133 & 0.054 & 1.45 & 59.7 & 38.84 & 0.0084 & tr. & 1.96 & 55.3 & 40.76 & 0.447 & 1.6 & 24 \\
\hline & 140 & 0.005 & 10.3 & 52.1 & 37.52 & 0.121 & 0.002 & 9.47 & 51.5 & 37.70 & 0.452 & 0.9 & 15 \\
\hline & 141 & 0.005 & 19.2 & 53.6 & 27.28 & 0.115 & tr. & 18.1 & 53.1 & 27.56 & 0.276 & 0.9 & 10 \\
\hline & 142 & 0.005 & 12.3 & 58.9 & 28.81 & 0.0242 & tr. & 12.2 & 55.9 & 30.44 & 0.301 & 1.2 & 13 \\
\hline & 143 & 0.005 & 19.9 & 57.8 & 22.34 & 0.0363 & tr. & 19.3 & 56.1 & 23.32 & 0.241 & 1.1 & 11 \\
\hline \multirow{4}{*}{1350} & 147 & 1.10 & - & 24.2 & 75.78 & 0.989 & 0.373 & 0.0138 & 25.4 & 73.21 & 0.662 & 0.7 & 119 \\
\hline & 148 & 1.10 & - & 41.2 & 58.79 & 0.957 & 0.065 & 0.0143 & 37.5 & 61.00 & 0.458 & 1.0 & 58 \\
\hline & 149 & 0.552 & - & 50.2 & 49.79 & 0.458 & 0.005 & 0.162 & 48.1 & 50.55 & 0.427 & 0.8 & 15 \\
\hline & 150 & 0.552 & - & 59.8 & 40.18 & 0.211 & 0.003 & 2.56 & 55.4 & 41.10 & 0.220 & 0.7 & 6 \\
\hline \multirow{7}{*}{1250} & & & & & & & & & & & $\left(\mathrm{Cu}_{2} \mathrm{O}\right)$ & & \\
\hline & 83 & 0.098 & 1.90 & 27.4 & 70.66 & 0.171 & - & 1.00 & 27.6 & 70.17 & 0.495 & 0.7 & 60 \\
\hline & 84 & 0.098 & 1.79 & 41.5 & 56.68 & 0.168 & - & 1.09 & 39.9 & 57.97 & 0.169 & 0.9 & 61 \\
\hline & 85 & 0.098 & 1.91 & 51.2 & 46.84 & 0.0585 & - & 2.25 & 48.6 & 47.81 & 0.098 & 1.2 & 30 \\
\hline & 86 & 0.098 & 1.91 & 46.2 & 51.88 & 0.123 & - & 1.65 & 44.4 & 52.45 & 0.138 & 1.4 & 60 \\
\hline & 87 & 0.098 & 1.91 & 46.2 & 51.88 & 0.104 & - & 1.79 & 45.4 & 51.90 & 0.146 & 0.8 & 25 \\
\hline & 88 & 0.098 & 2.08 & 60.3 & 37.63 & 0.00142 & - & 2.96 & 56.3 & 39.08 & 0.073 & 1.6 & 20 \\
\hline
\end{tabular}


Table 2. Experimental data for soda-based flux at $1250^{\circ} \mathrm{C}$. (wt\%)

\begin{tabular}{|c|c|c|c|c|c|c|c|c|c|c|c|c|}
\hline \multirow{2}{*}{ No. } & \multicolumn{4}{|c|}{ Initial } & \multicolumn{8}{|c|}{ Final } \\
\hline & $\underline{\mathbf{P}}$ & $\left(\mathrm{Na}_{2} \mathrm{O}\right)$ & $\left(\mathrm{SiO}_{2}\right)$ & $(\mathrm{CaO})$ & $\underline{P}$ & $\underline{\mathrm{Si}}$ & $\left(\mathrm{P}_{2} \mathrm{O}_{5}\right)$ & $\left(\mathrm{Na}_{2} \mathrm{O}\right)$ & $\left(\mathrm{SiO}_{2}\right)$ & $\left(\mathrm{Fe}_{t} \mathrm{O}\right)$ & $(\mathrm{CaO})$ & $\left(\mathrm{Al}_{2} \mathrm{O}_{3}\right)$ \\
\hline 134 & 0.552 & 25.5 & 50.43 & 24.1 & 0.464 & 0.012 & 0.0222 & 25.4 & 50.66 & 0.219 & 21.3 & 2.4 \\
\hline 135 & 0.552 & 33.4 & 50.39 & 16.2 & 0.463 & 0.006 & 0.0477 & 33.8 & 50.85 & 0.309 & 14.0 & 1.0 \\
\hline 136 & 0.552 & 41.8 & 50.15 & 8.03 & 0.458 & tr. & 0.164 & 42.6 & 50.25 & 0.392 & 5.80 & 0.7 \\
\hline 137 & 0.552 & 53.0 & 40.38 & 6.58 & 0.281 & 0.005 & 1.50 & 50.7 & 40.81 & 0.296 & 6.14 & 0.6 \\
\hline 138 & 0.552 & 47.5 & 40.49 & 12.0 & 0.350 & tr. & 1.34 & 46.2 & 40.74 & 0.257 & 10.9 & 0.5 \\
\hline 139 & 0.552 & 41.9 & 40.27 & 17.8 & 0.416 & tr. & 0.678 & 40.3 & 40.38 & 0.219 & 17.4 & 1.0 \\
\hline 621 & 0.552 & 56.2 & 37.77 & 6.0 & 0.128 & - & 4.30 & 51.6 & 37.47 & 0.168 & 6.46 & - \\
\hline \multirow[t]{2}{*}{622} & 0.552 & 52.8 & 35.36 & 12.0 & 0.103 & - & 4.39 & 47.7 & 34.93 & 0.135 & 12.8 & - \\
\hline & & & & $\left(\mathrm{CaF}_{2}\right)$ & & & & & & & & $\left(\mathrm{CaF}_{2}\right)$ \\
\hline 623 & 0.552 & 53.8 & 36.16 & 10.0 & 0.341 & - & 1.86 & 50.9 & 35.89 & 0.144 & 0.19 & 10.8 \\
\hline 624 & 0.552 & 47.8 & 32.14 & 20.0 & 0.463 & - & 0.932 & 45.9 & 33.52 & 0.114 & 0.33 & 19.2 \\
\hline
\end{tabular}

Table 4. Experimental data for lime-based flux.

\begin{tabular}{|c|c|c|c|c|c|c|c|c|c|}
\hline $\begin{array}{c}\text { Temp. } \\
\left({ }^{\circ} \mathbf{C}\right)\end{array}$ & No. & $\left(\frac{\mathrm{P}}{\%}\right)$ & $\stackrel{\underline{S}}{(p-m)}$ & $\begin{array}{c}\mathrm{P}_{2} \mathrm{O}_{5} \\
(\mathrm{ppm})\end{array}$ & $\begin{array}{c}\mathrm{S} \\
(\%)\end{array}$ & $\frac{\mathrm{CaF}_{2}}{(\%)}$ & $\begin{array}{l}\mathrm{CaO} \\
(\%)\end{array}$ & $\begin{array}{c}\mathrm{Al}_{2} \mathrm{O}_{3} \\
(\%)\end{array}$ & $\begin{array}{c}\mathrm{Fe}_{t} \mathrm{O} \\
(\mathrm{ppm})\end{array}$ \\
\hline \multirow{8}{*}{1430} & 706 & 0.547 & - & 8.9 & - & 99.3 & 0.66 & - & 41 \\
\hline & 707 & 0.556 & - & 24.4 & - & 90.1 & 9.86 & - & 47 \\
\hline & 708 & 0.578 & - & 68.3 & - & 82.2 & 17.8 & - & 82 \\
\hline & 721 & 0.594 & - & 9.2 & - & 80.6 & 9.50 & 9.87 & 63 \\
\hline & 722 & 0.544 & - & 11.4 & - & 71.2 & 17.5 & 11.2 & 80 \\
\hline & 723 & 0.573 & - & 63.1 & - & 59.3 & 29.6 & 11.1 & 97 \\
\hline & 724 & 0.590 & - & 9.7 & - & 59.0 & 18.5 & 22.5 & 97 \\
\hline & 725 & 0.547 & - & 11.1 & - & 50.8 & 27.4 & 21.8 & 107 \\
\hline \multirow{18}{*}{1500} & 332 & 0.554 & - & 12.4 & - & 90.1 & 9.92 & - & 48 \\
\hline & 333 & 0.533 & - & 44.8 & - & 79.7 & 20.3 & - & 126 \\
\hline & 334 & 0.534 & - & 40.8 & - & 72.8 & 27.2 & - & 164 \\
\hline & 401 & 0.529 & 20 & 16.9 & 1.78 & - & 44.0 & 54.1 & 1770 \\
\hline & 403 & 0.517 & 20 & 9.7 & 2.00 & 10.2 & 41.8 & 46.0 & 614 \\
\hline & 404 & 0.505 & 12 & 6.2 & 1.78 & 19.8 & 37.5 & 40.9 & 280 \\
\hline & 405 & 0.520 & 11 & 5.9 & 1.72 & 38.4 & 28.5 & 31.4 & 159 \\
\hline & 651 & 0.548 & - & 5.9 & - & 98.9 & 1.09 & - & 37 \\
\hline & 652 & 0.551 & - & 4.4 & - & 99.4 & 0.62 & - & 31 \\
\hline & 653 & 0.562 & - & 15.0 & - & 90.5 & 9.50 & - & 48 \\
\hline & 654 & 0.581 & - & 53.5 & - & 80.9 & 19.0 & - & 132 \\
\hline & 655 & 0.573 & - & 43.7 & - & 72.9 & 27.1 & - & 118 \\
\hline & 681 & 0.545 & - & 11.3 & - & 45.2 & 32.4 & 22.4 & 123 \\
\hline & 682 & 0.566 & - & 7.8 & - & 57.6 & 19.4 & 23.0 & 104 \\
\hline & 683 & 0.592 & - & 6.6 & - & 63.6 & 14.9 & 21.5 & 97 \\
\hline & 684 & 0.572 & - & 3.0 & - & 70.2 & 9.74 & 20.1 & 70 \\
\hline & 686 & 0.584 & - & 6.6 & - & 79.1 & 10.5 & 10.4 & 61 \\
\hline & 687 & 0.568 & - & 9.0 & - & 70.9 & 18.5 & 10.6 & 75 \\
\hline \multirow{8}{*}{1550} & 701 & 0.547 & - & 2.2 & - & 97.8 & 2.16 & - & 39 \\
\hline & 702 & 0.554 & - & 7.7 & - & 89.6 & 10.4 & - & 48 \\
\hline & 703 & 0.537 & - & 29.2 & - & 70.9 & 29.1 & - & 167 \\
\hline & 711 & 0.555 & - & 4.8 & - & 80.3 & 9.35 & 10.3 & 70 \\
\hline & 712 & 0.582 & - & 6.6 & - & 71.2 & 17.8 & 11.0 & 82 \\
\hline & 713 & 0.553 & - & 20.0 & - & 61.4 & 27.9 & 10.6 & 92 \\
\hline & 714 & 0.560 & - & 4.8 & - & 59.8 & 18.3 & 21.8 & 116 \\
\hline & 715 & 0.570 & - & 7.1 & - & 51.0 & 26.2 & 22.8 & 149 \\
\hline
\end{tabular}


Table 3. Experimental data for soda-based flux at $1250^{\circ} \mathrm{C}$. (wt $\%$ )

\begin{tabular}{|c|c|c|c|c|c|c|c|c|c|c|c|c|c|c|}
\hline \multirow{2}{*}{ No. } & \multicolumn{5}{|c|}{ Initial } & \multicolumn{9}{|c|}{ Final } \\
\hline & $\underline{\mathbf{P}}$ & $\underline{\mathrm{S}}$ & $\left(\mathrm{Na}_{2} \mathrm{O}\right)$ & $\left(\mathrm{SiO}_{2}\right)$ & & $\underline{P}$ & $\underline{\mathrm{S}}$ & $\left(\mathrm{P}_{2} \mathrm{O}_{5}\right)$ & (S) & $\left(\mathrm{Na}_{2} \mathrm{O}\right)$ & $\left(\mathrm{SiO}_{2}\right)$ & $\left(\mathrm{Fe}_{t} \mathrm{O}\right)$ & $\left(\mathrm{Al}_{2} \mathrm{O}_{3}\right)$ & \\
\hline 321 & 0.552 & 0.499 & 50.8 & 49.23 & & 0.467 & 0.0097 & 0.491 & 1.76 & 47.4 & 48.40 & 1.27 & 0.7 & \\
\hline \multirow[t]{2}{*}{322} & 0.545 & 0.985 & 60.5 & 39.53 & & 0.270 & 0.0031 & 2.26 & 3.29 & 54.7 & 38.18 & 0.872 & 0.7 & \\
\hline & & & & & $(\mathrm{BaO})$ & & & & & & & & & $(\mathrm{BaO})$ \\
\hline 301 & 0.552 & 0.499 & 42.7 & 46.58 & 10.8 & 0.486 & 0.0138 & 0.315 & 1.75 & 41.6 & 44.90 & 1.30 & 0.8 & 9.38 \\
\hline 302 & 0.552 & 0.499 & 35.1 & 43.36 & 21.5 & 0.497 & 0.0179 & 0.191 & 1.76 & 34.2 & 42.51 & 1.19 & 0.7 & 19.41 \\
\hline 303 & 0.552 & 0.499 & 27.6 & 40.14 & 32.3 & 0.538 & 0.0213 & 0.105 & 1.77 & 27.8 & 39.17 & 0.953 & 0.9 & 29.35 \\
\hline 306 & 0.545 & 0.985 & 54.5 & 37.59 & 7.9 & 0.383 & 0.0040 & 1.93 & 4.53 & 47.2 & 35.08 & 1.24 & 0.6 & 9.43 \\
\hline 307 & 0.545 & 0.985 & 48.5 & 35.65 & 15.9 & 0.403 & 0.0049 & 1.44 & 3.94 & 42.6 & 34.70 & 1.03 & 0.5 & 15.84 \\
\hline \multirow[t]{2}{*}{308} & 0.545 & 0.985 & 42.4 & 33.83 & 23.8 & 0.414 & 0.0056 & 1.39 & 3.41 & 39.5 & 33.29 & 0.822 & 0.6 & 21.00 \\
\hline & & & & & $(\mathrm{MgO})$ & & & & & & & & & $(\mathrm{MgO})$ \\
\hline 311 & 0.552 & 0.499 & 42.7 & 51.33 & 6.0 & 0.510 & 0.0166 & 0.110 & 1.69 & 42.1 & 48.93 & 0.818 & 0.4 & 5.93 \\
\hline 312 & 0.552 & 0.499 & 35.1 & 52.86 & 12.0 & 0.569 & 0.0290 & 0.0435 & 1.62 & 34.9 & 50.70 & 0.546 & 0.6 & 11.62 \\
\hline 313 & 0.552 & 0.499 & 27.6 & 54.27 & 18.0 & 0.572 & 0.0647 & 0.0321 & 1.40 & 28.8 & 51.75 & 0.466 & 0.3 & 17.25 \\
\hline 316 & 0.545 & 0.985 & 54.5 & 40.46 & 5.0 & 0.365 & 0.0047 & 1.38 & 3.22 & 49.5 & 39.09 & 0.896 & 0.4 & 5.54 \\
\hline 317 & 0.545 & 0.985 & 48.5 & 41.52 & 10.0 & 0.426 & 0.0078 & 0.748 & 3.49 & 46.0 & 40.34 & 1.10 & 0.6 & 7.71 \\
\hline 318 & 0.545 & 0.985 & 42.4 & 42.58 & 15.0 & 0.480 & 0.0164 & 0.329 & 3.66 & 42.3 & 44.26 & 2.14 & 0.6 & 6.68 \\
\hline
\end{tabular}

Table 5. Experimental data for fluoride-based flux.

\begin{tabular}{|c|c|c|c|c|c|c|c|}
\hline No. & $\begin{array}{c}\text { Temp. } \\
\left({ }^{\circ} \mathrm{C}\right)\end{array}$ & $\left(\frac{\mathrm{P}}{\%)}\right.$ & $\begin{array}{c}\mathrm{P}_{2} \mathrm{O}_{5} \\
(\mathrm{ppm})\end{array}$ & $\begin{array}{l}\mathrm{BaF}_{2} \\
(\%)\end{array}$ & $\begin{array}{l}\mathrm{BaO} \\
(\%)\end{array}$ & $\begin{array}{r}\mathrm{Fe}_{t} \mathrm{O} \\
(\mathrm{ppm})\end{array}$ & \\
\hline 386 & 1350 & 0.519 & 62.8 & 94.9 & 5.1 & 78 & \\
\hline 387 & 1350 & 0.468 & $2.01 \times 10^{3}$ & 86.8 & 13.0 & 118 & \\
\hline 388 & 1350 & 0.295 & $1.96 \times 10^{4}$ & 72.9 & 25.0 & 187 & \\
\hline 389 & 1350 & 0.251 & $1.52 \times 10^{4}$ & 76.2 & 22.3 & 153 & \\
\hline 660 & 1350 & 0.503 & 8.2 & 100.0 & - & 58 & \\
\hline 661 & 1350 & 0.548 & 8.8 & 99.5 & 0.5 & 65 & \\
\hline 662 & 1350 & 0.538 & 7.2 & 100.0 & - & 60 & \\
\hline 663 & 1350 & 0.709 & 500 & 90.9 & 9.0 & 90 & \\
\hline \multirow[t]{2}{*}{664} & 1350 & 0.368 & $1.98 \times 10^{3}$ & 82.3 & 17.5 & 135 & \\
\hline & & & & $\left(\mathrm{MgF}_{2}\right)$ & $(\mathrm{MgO})$ & & \\
\hline 372 & 1500 & 0.527 & 2.3 & 92.9 & 7.1 & 74 & \\
\hline 666 & 1350 & 0.617 & 1.5 & 98.6 & 1.4 & 61 & \\
\hline 667 & 1350 & 0.523 & 1.2 & 99.3 & 0.68 & 56 & \\
\hline \multirow[t]{2}{*}{668} & 1350 & 0.531 & 4.5 & 93.8 & 6.2 & 85 & \\
\hline & & & & $(\mathrm{NaF})$ & $\left(\mathrm{Na}_{2} \mathrm{O}\right)$ & & \\
\hline 671 & 1350 & 0.547 & 112 & 98.0 & 2.0 & 568 & \\
\hline \multirow[t]{2}{*}{672} & 1350 & 0.535 & 22.4 & 99.4 & 0.6 & 430 & \\
\hline & & & & $\left(\mathrm{CaF}_{2}\right)$ & $(\mathrm{BaO})$ & & $(\mathrm{CaO})$ \\
\hline 351 & 1500 & 0.494 & 8.2 & 89.8 & 9.9 & 32 & 0.3 \\
\hline 352 & 1500 & 0.565 & 13.3 & 79.8 & 19.6 & 37 & 0.7 \\
\hline \multirow[t]{2}{*}{353} & 1500 & 0.541 & 14.7 & 71.6 & 28.4 & 41 & - \\
\hline & & & & $\left(\mathrm{CaF}_{2}\right)$ & $(\mathrm{MgO})$ & & $(\mathrm{GaO})$ \\
\hline 361 & 1500 & 0.533 & 8.2 & 89.3 & 10.5 & 56 & 0.2 \\
\hline 362 & 1500 & 0.550 & 12.9 & 78.6 & 21.0 & 170 & 0.4 \\
\hline \multirow[t]{2}{*}{363} & 1500 & 0.545 & 15.3 & 69.2 & 30.5 & 273 & 0.3 \\
\hline & & & & $\left(\mathrm{BaF}_{2}\right)$ & $(\mathrm{CaO})$ & & $(\mathrm{BaO})$ \\
\hline 396 & 1350 & 0.512 & 73.6 & 92.0 & 8.0 & 140 & - \\
\hline 397 & 1350 & 0.493 & 159 & 83.4 & 16.6 & 166 & - \\
\hline
\end{tabular}

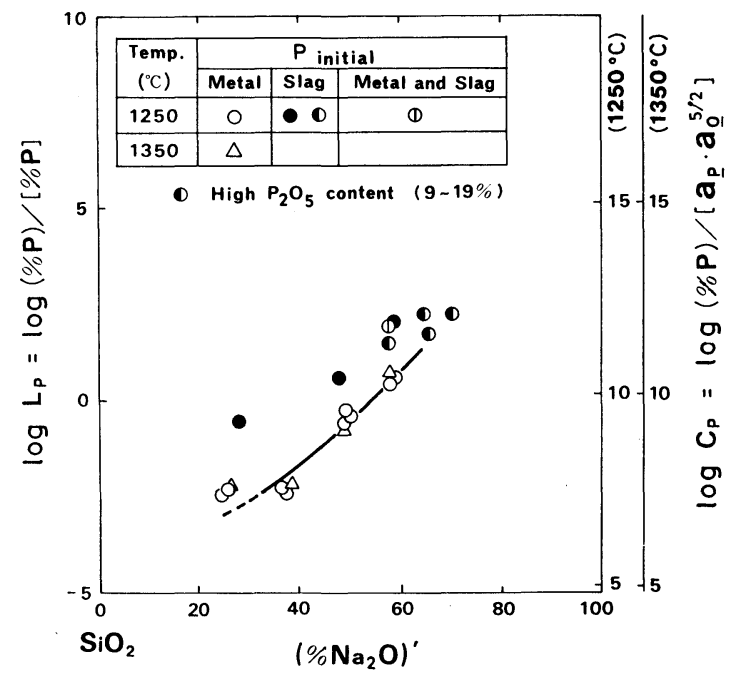

Fig. 1. Logarithms of $L_{\mathrm{P}}\left(C_{\mathrm{P}}\right)$ in the $\mathrm{Na}_{2} \mathrm{O}-\mathrm{SiO}_{2}$ system at 1250 and $1350^{\circ} \mathrm{C}$.

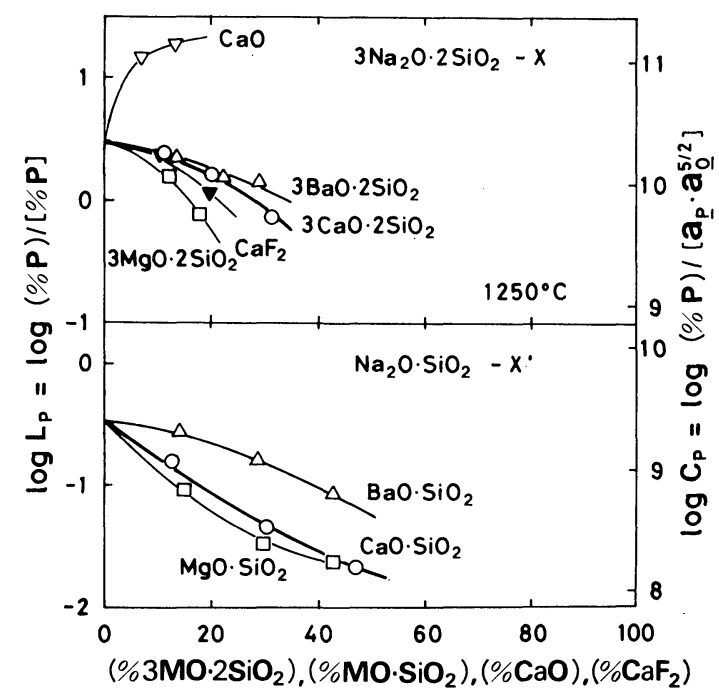

Fig. 2. Logarithms of $L_{\mathrm{P}}\left(C_{\mathrm{P}}\right)$ in the $3 \mathrm{Na}_{2} \mathrm{O} \cdot 2 \mathrm{SiO}_{2}-3 \mathrm{MO}$. $2 \mathrm{SiO}_{2}$ and $\mathrm{Na}_{2} \mathrm{O} \cdot \mathrm{SiO}_{2}-\mathrm{MO} \cdot \mathrm{SiO}_{2}(\mathrm{M}=\mathrm{Mg}, \mathrm{Ca}$, Ba) pseudo-binary systems at $1250^{\circ} \mathrm{C}$. 


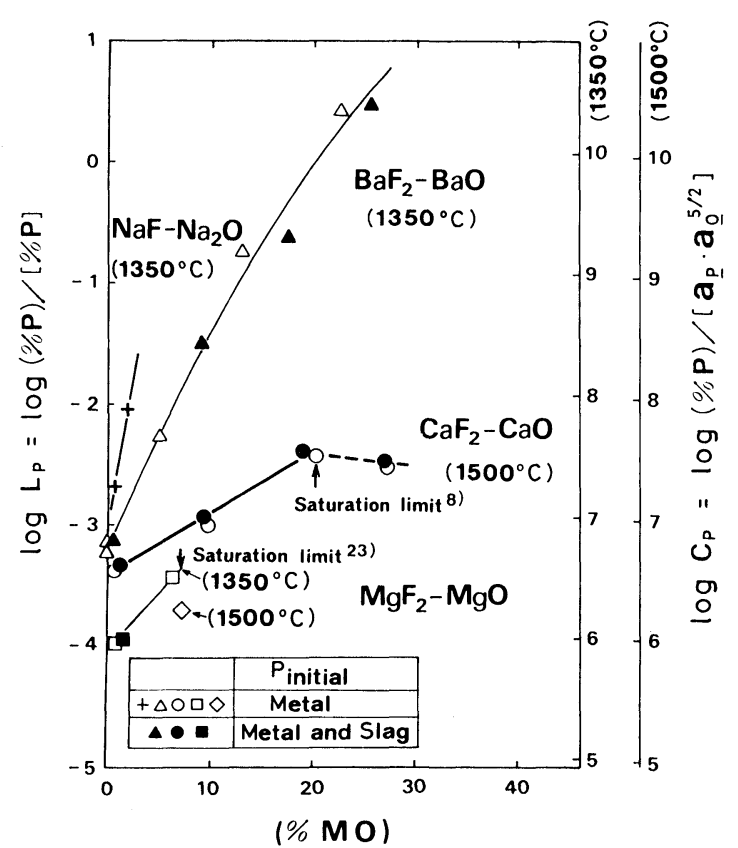

Fig. 3. Logarithms of $L_{\mathrm{P}}\left(C_{\mathrm{P}}\right)$ in the $\mathrm{MF}_{2}(\mathrm{MF})-\mathrm{MO}\left(\mathrm{M}_{2} \mathrm{O}\right)$ $(\mathrm{M}=\mathrm{Na}, \mathrm{Ba}, \mathrm{Ca}, \mathrm{Mg})$ system.

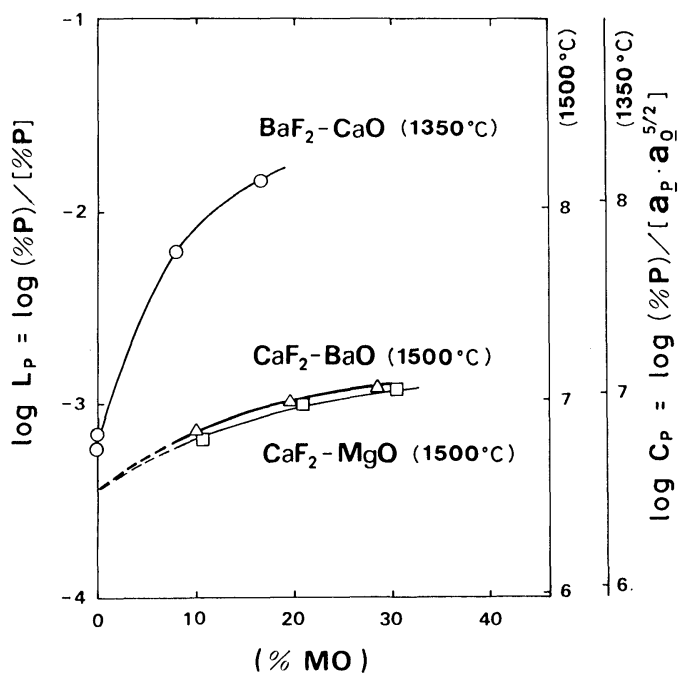

Fig. 4. Logarithms of $L_{\mathrm{P}}\left(C_{\mathrm{P}}\right)$ in the $\mathrm{BaF}_{2}-\mathrm{CaO}, \mathrm{CaF}_{2}-\mathrm{BaO}$ and $\mathrm{CaF}_{2}-\mathrm{MgO}$ systems.

\section{Discussion}

\section{Phosphorus Equilibrium between Slag and Metal}

Slag-metal equilibria concerning phosphorus can be expressed in terms of molecular and ionic forms as follows;

$$
\begin{aligned}
& \underline{\mathrm{P}}+5 / 2 \underline{\mathrm{O}}=\mathrm{PO}_{2.5} \\
& \underline{\mathrm{P}}+5 / 2 \underline{\mathrm{O}}+3 / 2\left(\mathrm{O}^{2-}\right)=\left(\mathrm{PO}_{4}^{3-}\right)
\end{aligned}
$$

where the equilibrium constants for Eqs. (1) and (2) are now represented by $K_{\mathrm{P}}$ and $K_{\text {ion }}$, respectively.

$$
\begin{aligned}
& K_{\mathrm{P}}=\mathcal{N}_{\mathrm{PO}_{2.5}} \cdot \gamma_{\mathrm{PO}_{2.5}} /\left[a_{\underline{\mathrm{P}}} \cdot a_{\underline{\mathrm{O}}}^{5 / 2}\right] \\
& K_{\text {ion }}=a_{\mathrm{PO}_{4}^{3-}} /\left[a_{\underline{\mathrm{P}}} \cdot a_{\underline{\underline{0}}}^{5 / 2} \cdot a_{\mathrm{O}^{2}}^{3 / 2}\right]
\end{aligned}
$$

From Eqs. (3) and (4), we obtain

$$
\begin{aligned}
& (\% \mathrm{P}) /\left[a_{\underline{\mathrm{P}}} \cdot a_{\underline{0}}^{5 / 2}\right]=\kappa_{\mathrm{P}} / \gamma_{\mathrm{PO}_{2.5}} \times(\% \mathrm{P}) / \mathcal{N}_{\mathrm{PO}_{2.5}} \\
& (\% \mathrm{P}) /\left[a_{\mathrm{P}} \cdot a_{\underline{0}}^{5 / 2}\right]=\kappa_{\mathrm{ion}} / \gamma_{\mathrm{PO}_{4}^{3-}} \times(\% \mathrm{P}) / \mathcal{N}_{\mathrm{PO}_{4}^{3-}}
\end{aligned}
$$

where $\mathcal{N}_{\mathrm{PO}_{4}^{3-}}^{3-}$ denotes the anionic fraction of $\mathrm{PO}_{4}^{3-}$ ion and $a_{i}, \gamma_{i}$ and $\mathcal{N}_{i}$ represent their usual meanings.

It should be noted that the activities, and the activity coefficients of ionic species as well as the equilibrium constant, $K_{\text {ion }}$ cannot be experimentally determined and cannot be calculated without nonthermodynamic assumptions. The left hand side of Eq. (5) or Eq. (6) is now defined by phosphate capacity, $C_{\mathrm{P}}$. Provided that the terms (\% $\left.\mathrm{P}\right) / \mathcal{N}_{\mathrm{PO}_{2.5}}$ and $(\% \mathrm{P}) /$ $\mathcal{N}_{\mathrm{PO}_{4}^{3-}}$ in Eqs. (5) and (6) are independent of slag compositions at a constant temperature, the phosphate capacity is inversely proportional to the activity coefficients of $\mathrm{PO}_{2.5}\left(\mathrm{PO}_{4}^{3-}\right)$. The following relationship concerning $C_{\mathrm{P}}, L_{\mathrm{P}}$ and $a_{\mathrm{O}}$ can be obtained as

$$
L_{\mathrm{P}}=C_{\mathrm{P}} \cdot a_{\underline{\mathrm{o}}}^{5 / 2} \cdot f_{\mathrm{P}}
$$

where $f_{\mathrm{P}}$ denotes the activity coefficient of phosphorus in metal which can be obtained in the present work from the values for $e_{\mathrm{P}}^{(\mathrm{P})}=0.062,{ }^{12)} \quad e_{\mathrm{P}}^{(\mathrm{C})}=0.13,{ }^{13)} e_{\mathrm{P}}^{(\mathrm{S})}=$ $0.028^{12)}$ and $e_{\mathrm{P}}^{(\mathrm{Si})}=0.12^{12)}$ as well as the value ${ }^{14)}$ for $[\% \mathrm{C}]_{\text {sat. }}=1.30+2.57 \times 10^{-3} \cdot \mathrm{T}\left({ }^{\circ} \mathrm{C}\right)-0.33[\% \mathrm{P}]-0.36$ $[\% \mathrm{~S}]-0.317[\% \mathrm{Si}]$. In this work, the second order interaction coefficient $\left(r_{\mathbf{P}}^{(\mathrm{C})}\right)^{13)}$ has not been used for

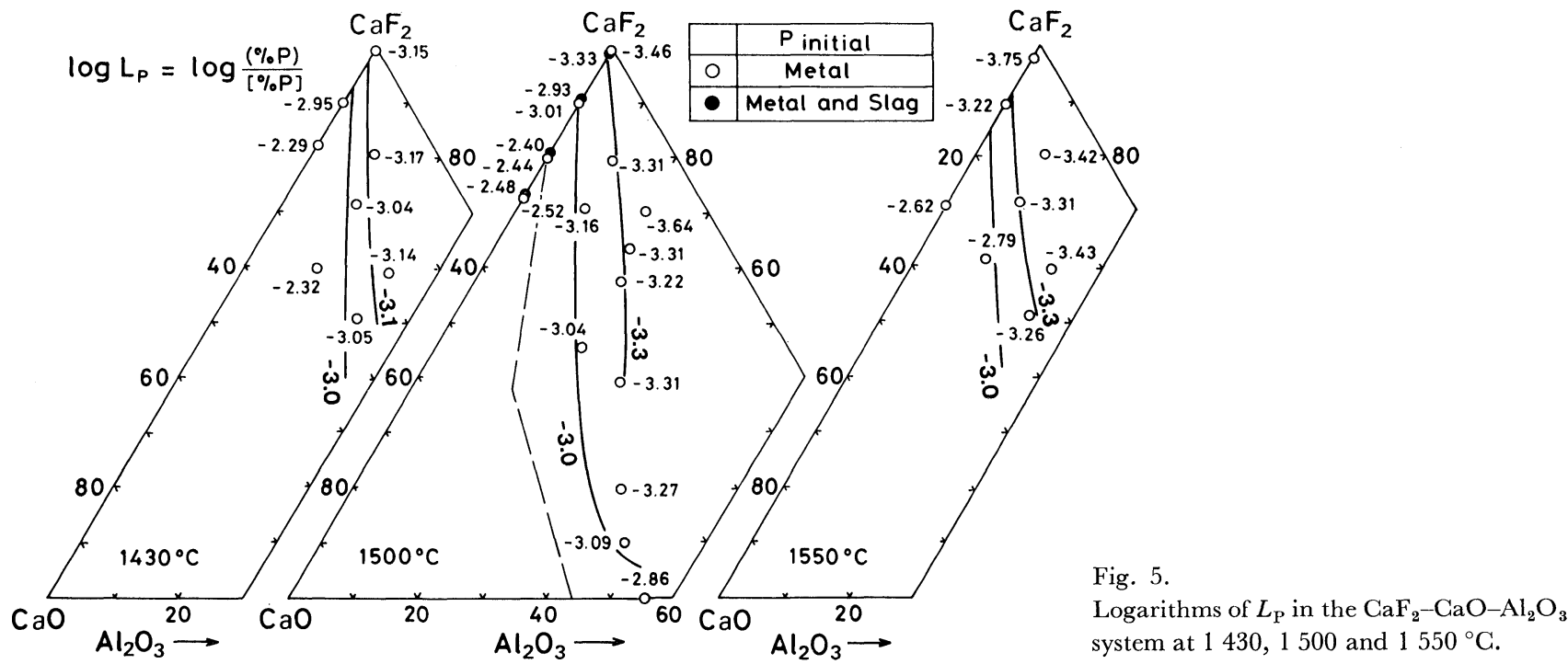


the calculation of $f_{\mathrm{P}}$, although its effect on $\log C_{\mathrm{P}}$ is about 0.3 at maximum. The value of $a_{0}$ under the present experimental conditions, i.e., $a_{\mathrm{C}}=1, P_{\mathrm{CO}}=1 \mathrm{~atm}$ can be obtained from the standard free-energy change $\Delta G^{\circ}$ for the reaction:

$$
\mathrm{C}+\underline{\mathrm{O}}=\mathrm{CO} \quad \Delta G^{\circ}=1300-20.26 T^{12,15)}(\mathrm{cal})
$$

The values of $C_{\mathrm{P}}$ obtain by substituting the value of $a_{\mathrm{o}}$ at corresponding temperatures into Eq. (7) are also shown in Figs. 1 to 4 . The value for $C_{\mathrm{P}}$ which is the experimentally obtainable quantity is not only a function of slag compositions and temperatures, but also is the value only limited to a liquid iron-slag system. The value of $C_{\mathrm{PO}_{4}^{3-}}\left(=\%\left(\mathrm{PO}_{4}^{3-}\right) /\left[P_{\mathrm{P}_{2}}^{1 / 2} \cdot P_{\mathrm{O}_{2}}^{5 / 4}\right]\right)$ defined by Wagner ${ }^{16)}$ is only a function of slag compositions at a given temperature. The present authors, however, recommend to use $C_{\mathrm{P}}$ rather than $G_{\mathrm{PO}_{4}^{3-}}$, since it is more convenient to use $G_{\mathrm{P}}$ rather than $C_{\mathrm{PO}_{4}^{3-}}^{3-}$ when compared with the plant data. ${ }^{5)}$ By use of the free-energy change for the following reactions, the relation between $C_{\mathrm{P}}$ and $C_{\mathrm{PO}_{4}^{3-}}$ is calculated and given by Eq. (11).

$$
\begin{aligned}
1 / 2 \mathrm{P}_{2} & =\underline{\mathrm{P}}_{\mathrm{in} \mathrm{Fe}} \\
\Delta G^{\circ} & =-29200-4.6 T^{12)} \quad(\mathrm{cal}) \\
1 / 2 \mathrm{O}_{2} & =\underline{\mathrm{O}}_{\mathrm{in} \mathrm{Fe}} \\
\Delta G^{\circ} & =-28000-0.69 T^{12)} \quad(\mathrm{cal}) \\
\log C_{\mathrm{P}} & =\log C_{\mathrm{PO}_{4}^{3-}}-21680 / T-1.87
\end{aligned}
$$

\section{Phosphate Capacity $C_{\mathrm{P}}$ in Soda- and Lime-based Fluxes}

\section{Soda-based Fluxes}

As shown in Fig. 1, the values for $L_{\mathrm{P}}$ in the $\mathrm{Na}_{2} \mathrm{O}-$ $\mathrm{SiO}_{2}$ slags obtained by approaching the phosphorus equilibrium from the slag side are greater than those from the metal side. This trend is not observed in the $\mathrm{CaF}_{2}-\mathrm{CaO}$ system shown in Fig. 3, but observed only in the $\mathrm{Na}_{2} \mathrm{O}-\mathrm{SiO}_{2}$ system. It was found in the $\mathrm{Na}_{2} \mathrm{O}-\mathrm{SiO}_{2}$ slags that the sulfur ${ }^{6)}$ and manganese ${ }^{17)}$ distribution ratios approached from the metal side agreed well with those from the slag side. Th results ${ }^{7)}$ for $C_{\mathrm{S}}$ obtained from the sulfur distribution ratios between the $\mathrm{CaF}_{2}-\mathrm{CaO}-\mathrm{Al}_{2} \mathrm{O}_{3}$ slags and carbon-saturated iron melts at $1500{ }^{\circ} \mathrm{C}$ are in good agreement with those from the gas-slag equilibrium experiments. ${ }^{8)}$ Smith and Davies ${ }^{18)}$ obtained the activity coefficient of $\mathrm{MnO}$ from the manganese distribution equilibrium between the $\mathrm{CaF}_{2}-\mathrm{CaO}$ slags and a solid $\mathrm{Pt}$. The manganous capacity, $C_{\mathrm{Mn}}$ $\left(=(\% \mathrm{Mn}) /\left[a_{\mathrm{Mn}} \cdot a_{\mathrm{O}}\right]\right)$ calculated from their results was found to be in agreement with that obtained from the manganese distribution equilibrium between the $\mathrm{CaF}_{2}-\mathrm{CaO}$ slags and carbon-saturated iron melts. ${ }^{17)}$ These experimental findings thus suggest that the oxygen potentials in the experiments of sulfur and manganese partitions are undoubtedly controlled by the $\mathrm{C}\left(a_{\mathrm{C}}=1\right)-\mathrm{CO}\left(P_{\mathrm{CO}}=1 \mathrm{~atm}\right)$ equilibrium. It can be seen from Fig. 1 that no apparent difference between $L_{\mathrm{P}}$ obtained at $1250^{\circ} \mathrm{C}$ and that at $1350^{\circ} \mathrm{C}$ was observed.

As listed in Table 1, the phosphorus content in the carbon-saturated copper melts equilibrated with the $\mathrm{Na}_{2} \mathrm{O}-\mathrm{SiO}_{2}$ fluxes is less than $0.2 \mathrm{wt} \%$ and the carbon content is also extremely low $(<10 \mathrm{ppm})$. The activity of phosphorus in the carbon-saturated copper melt, $a_{\mathrm{P}}$ in $\mathrm{Cu}$ therefore is considered to be equal to the phosphorus content $[\% \mathrm{P}]$. The activities of $\mathrm{Cu}$ in the $\mathrm{Cu}-\mathrm{P}$ alloy were calculated at $1200^{\circ} \mathrm{C}$ by $\mathrm{Kramss}{ }^{19)}$ from the phase diagram of the $\mathrm{Cu}-\mathrm{P}$ system by assuming the regular solution. By use of the Gibbs-Duhem relationship, the activity coefficient of phosphorus with respect to the Raoultian standard state, $\gamma_{P}^{\circ}$ was obtained as 0.025. From this value and the regular solution approximation, the free-energy change for the following reaction was derived as

$$
\begin{aligned}
1 / 2 \mathrm{P}_{2} & =\underline{\mathrm{P}}_{\text {in Cu }} \\
\Delta G^{\circ} & =-10800-7.72 T(\mathrm{cal})
\end{aligned}
$$

It can be seen from Eqs. (9) and (12) that the activity of phosphorus in iron is 91 times as high as that in copper at $1250^{\circ} \mathrm{C}$. The values for $C_{\mathrm{P}}$ obtained in the copper melts were converted to those for $C_{\mathrm{P}}$ in the carbon-saturated iron melts by use of the above relation. These results are given in Fig. 6 , indicating the comparatively good agreement with those obtained in the carbon-saturated iron melts.

From the results on the phosphorus equilibrium between the $\mathrm{Na}_{2} \mathrm{O}-\mathrm{SiO}_{2}-\mathrm{PO}_{2.5}-\mathrm{Fe}_{t} \mathrm{O}$ slags and an iron foil at $1300^{\circ} \mathrm{C}$, Ito and $\mathrm{Sano}^{20)}$ obtained the phosphorus partitions with respect to a carbonsaturated iron melt by calculation and the dependence of the $C_{\mathrm{PO}_{4}^{3-}}$ value on slag compositions is given by use of the activities of $\mathrm{Fe}_{t} \mathrm{O}^{21)}$ in $\mathrm{Na}_{2} \mathrm{O}-\mathrm{SiO}_{2}-\mathrm{PO}_{2.5}$ $\mathrm{Fe}_{t} \mathrm{O}-\mathrm{Na}_{2} \mathrm{CO}_{3}$ system. The contents of $\mathrm{PO}_{2.5}$ and $\mathrm{Fe}_{t} \mathrm{O}$ in their slags were in the range of $3 \sim 44 \mathrm{wt} \%$ and $2 \sim 33 \mathrm{wt} \%$, respectively. Although these values are higher than those in the present work $\left(\mathrm{Fe}_{t} \mathrm{O}\right.$ wt $\%$ $\leq 1.3)$, the values for $C_{\mathrm{P}}$ in their slags containing $\mathrm{Fe}_{t} \mathrm{O} \mathrm{wt}^{\mathrm{O}} \%=2 \sim 6\left(\mathcal{N}_{\mathrm{Fe}_{t} \mathrm{O}}=0.01 \sim 0.04\right)$ and $\mathrm{Fe}_{t} \mathrm{O} w \mathrm{t} \%$ $=10 \sim 14\left(\mathcal{N}_{\mathrm{Fe}_{t} \mathrm{O}}=0.09 \sim 0.12\right)$ were calculated by use

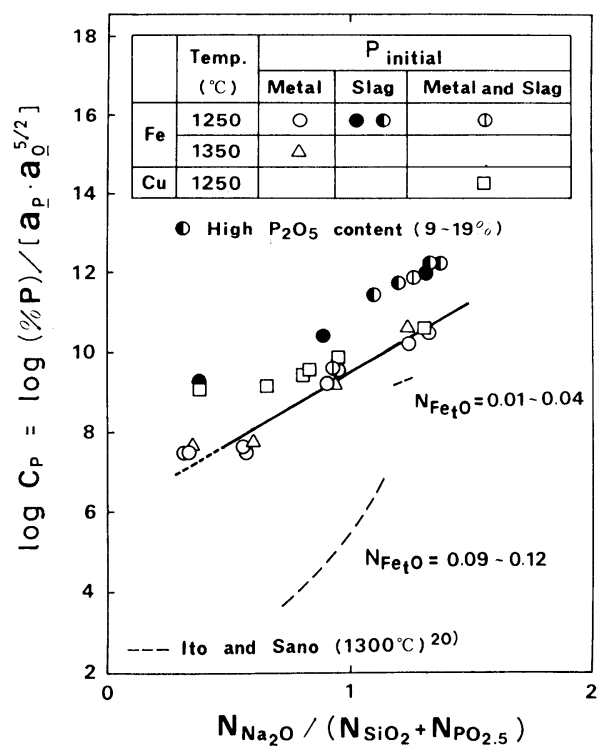

Fig. 6. Comparison of the present value for $\log C_{\mathrm{P}}$ with the previous results. ${ }^{201}$ 
of Eq. (11). The results are shown in Fig. 6 by a dotted line for comparison. One may see that their results tend to approach the present results with a decrease of the $\mathrm{Fe}_{t} \mathrm{O}$ content.

It is seen from Fig. 2 that when $\mathrm{Na}_{2} \mathrm{O}$ is replaced by $\mathrm{MgO}(\mathrm{CaO}, \mathrm{BaO})$ at a constant $\mathrm{SiO}_{2}$ content in the $\mathrm{Na}_{2} \mathrm{O}-\mathrm{SiO}_{2}-\mathrm{MgO}(\mathrm{CaO}, \mathrm{BaO})$ ternary system, $\log C_{\mathrm{P}}$ gradually decreases in the order of $\mathrm{Ba}>\mathrm{Ca}>$ $\mathrm{Mg}$. The fact that the replacement of $\mathrm{Na}_{2} \mathrm{O}$ by $\mathrm{BaO}$ lowers the value of $C_{\mathrm{P}}$ indicates that $\log C_{\mathrm{P}}$ in the pure liquid $\mathrm{Na}_{2} \mathrm{O}$ is greater than that in the pure $\mathrm{BaO}$.

The activity coefficients of $\mathrm{P}_{2} \mathrm{O}_{5}$ in the $\mathrm{Na}_{2} \mathrm{O}-\mathrm{P}_{2} \mathrm{O}_{5}$ system were calculated by using the Gibbs-Duhem equation from the activity coefficients of $\mathrm{Na}_{2} \mathrm{O}$ which were determined in the previous report. ${ }^{6)}$ The values for $\log C_{\mathrm{P}}$ were obtained from the activity coefficients of $\mathrm{P}_{2} \mathrm{O}_{5}$ and are shown in Fig. 7. The details of this derivation of $\log C_{\mathrm{P}}$ from $\gamma_{\mathrm{P}_{2} \mathrm{O}_{5}}$ are described elsewhere. ${ }^{4)}$ The phosphorus distribution ratios between the carbon-saturated iron melts and the $\mathrm{Na}_{2} \mathrm{O}-\mathrm{SiO}_{2}$ slags containing $9 \sim 19 \mathrm{wt} \% \mathrm{P}_{2} \mathrm{O}_{5}$ was studied by approaching the phosphorus equilibrium from the slag side. These results given in Table 1 (Nos. 140 143) and Fig. 1 are also shown in Figs. 6 and 7. As pointed out previously it appears that these values for $C_{\mathrm{P}}$ are considerably higher than those obtained by approaching the phosphorus equilibrium from the metal side.

Recently, Tsukihashi et al. ${ }^{22)}$ obtained the phosphorus distribution ratios between the $\mathrm{Na}_{2} \mathrm{O}(40 \sim$ $65 \%)-\mathrm{SiO}_{2}-\mathrm{P}_{2} \mathrm{O}_{5}(5 \sim 25 \%)$ slags and carbon-saturated iron melts at $1200{ }^{\circ} \mathrm{C}$. Their results are also shown in Fig. 7, indicating an agreement with those obtained in the present work. Judging from the values for $\log C_{\mathrm{P}}$ in the $\mathrm{Na}_{2} \mathrm{O}-\mathrm{P}_{2} \mathrm{O}_{5}$ system indicated in Fig. 7, the values for $\log C_{\mathrm{P}}$ in the $\mathrm{Na}_{2} \mathrm{O}-\mathrm{SiO}_{2}-\mathrm{P}_{2} \mathrm{O}_{5}$ system appear to be unreasonably too high, since these values are obtained by approaching the phosphorus equilibrium from the slag side.

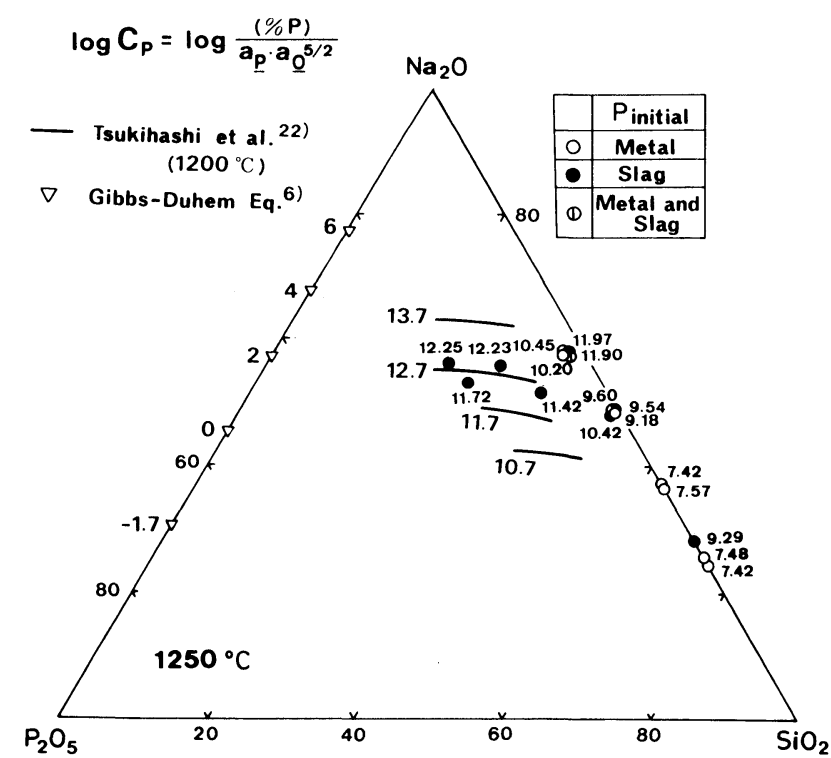

Fig. 7. Logarithms of $C_{\mathrm{P}}$ in the $\mathrm{Na}_{2} \mathrm{O}-\mathrm{SiO}_{2}-\mathrm{P}_{2} \mathrm{O}_{5}$ system at $1250^{\circ} \mathrm{C}$.

\section{Lime-based Fluxes}

It can be seen from Fig. 3 that the values for $\log C_{\mathrm{P}}$ in the fluoride-oxide system with common cations decrease in the order of $\mathrm{Na}>\mathrm{Ba}>\mathrm{Ca}>\mathrm{Mg}$. The chemical analysis for the pure fluorides indicated that the significant amount of oxide $\left(\mathcal{N}_{\mathrm{Na}_{2} \mathrm{O}}=0.014, \mathcal{N}_{\mathrm{BaO}}=\right.$ $\left.0.006, \mathcal{N}_{\mathrm{CaO}}=0.03, \mathcal{N}_{\mathrm{MgO}}=0.022\right)$ was observed, although the $\mathrm{CO}$ as well as Ar gas used for purging was dried by $\mathrm{P}_{2} \mathrm{O}_{5}$ before the introduction into the furnace. The compositions in the saturation limit for the system $\mathrm{CaF}_{2}-\mathrm{CaO}\left(\mathcal{N}_{\mathrm{CaO}}=0.26, \mathrm{CaO} \mathrm{wt}^{\circ} \%=20 \text { at } 1500{ }^{\circ} \mathrm{C}\right)^{8)}$ and $\mathrm{MgF}_{2}-\mathrm{MgO}$ system $\left(\mathcal{N}_{\mathrm{MgO}}=0.10, \mathrm{MgO}\right.$ w $\%=7$ at $\left.1350{ }^{\circ} \mathrm{C}\right)^{23)}$ are indicated in Fig. 3 by the arrows. The maximum values for the logarithms of $C_{\mathrm{P}}$ in the liquid region of the $\mathrm{CaF}_{2}-\mathrm{CaO}$ system at $1500{ }^{\circ} \mathrm{C}$ are roughly equal to those in the composition with the $\mathrm{Na}_{2} \mathrm{O} / \mathrm{SiO}_{2}=1 / 2$ (wt $\%$ ratio). On the other hand, $\log C_{\mathrm{S}}$ in the liquid region of the $\mathrm{CaF}_{2}-\mathrm{CaO}$ system ${ }^{8)}$ at $1500{ }^{\circ} \mathrm{C}$ are considerably higher than those in the $\mathrm{Na}_{2} \mathrm{O}-\mathrm{SiO}_{2}$ system $^{6)}$ at $1250{ }^{\circ} \mathrm{C}$.

It is apparent from Fig. 2 that the values for $\log C_{\mathrm{P}}$ increase or decrease with the addition of $\mathrm{CaO}$ or $\mathrm{CaF}_{2}$ to the melt with the $3 \mathrm{Na}_{2} \mathrm{O} \cdot 2 \mathrm{SiO}_{2}$ composition, respectively. The increase in $\log C_{\mathrm{P}}$ with the addition of $\mathrm{CaO}$ can be explained by the difference between the values for $\log C_{\mathrm{P}}$ at the $3 \mathrm{Na}_{2} \mathrm{O} \cdot 2 \mathrm{SiO}_{2}$ composition and that in the pure liquid $\mathrm{CaO} .{ }^{4)}$ Similarly, the decrease in $\log C_{\mathrm{P}}$ with the addition of $\mathrm{CaF}_{2}$ can also be explained by the difference between the value of $\log C_{\mathrm{P}}$ at the $3 \mathrm{Na}_{2} \mathrm{O} \cdot 2 \mathrm{SiO}_{2}$ composition and that in the pure liquid $\mathrm{CaF}_{2}$ at $1500{ }^{\circ} \mathrm{C}$. The logarithms of $C_{\mathrm{P}}$ are expected to increase as a result of the increase in oxygen ion followed by the fluorination reaction: $(\mathrm{Si}-\mathrm{O}-\mathrm{Si})+2 \mathrm{~F}^{-}=2(\mathrm{Si}-\mathrm{F})+\mathrm{O}^{2-}$, if fluoride such as $\mathrm{CaF}_{2}$ is added to the silicate melt with acidic compositions. This is confirmed by Bronson and St. Pierre $^{24)}$ in the experiment of the sulfide capacity measurement at $1503{ }^{\circ} \mathrm{C}$; that is, $\log C_{\mathrm{S}}$ was observed to increase with the addition of $\mathrm{CaF}_{2}$ (up to $10 \mathrm{wt} \%$ ) to the $\mathrm{CaO}-\mathrm{SiO}_{2}$ slags with the $\mathrm{CaO} / \mathrm{SiO}_{2}(\mathrm{wt} \%)=$ $1 \sim 1.28$. Actually, however, as the present slag composition $\left(3 \mathrm{Na}_{2} \mathrm{O} \cdot 2 \mathrm{SiO}_{2}\right)$ is basic, no increase in $\log C_{\mathrm{P}}$ was observed with the addition of $\mathrm{CaF}_{2}$.

The effect of $\mathrm{Na}_{2} \mathrm{O}$ or $\mathrm{CaF}_{2}$ on $\log C_{\mathrm{P}}$ on mole fraction basis has been studied. The results are shown in Fig. 8, where the $\mathrm{CaO}$ equivalencies of $\mathrm{Na}_{2} \mathrm{O}$ on the mole fraction basis obtained from the data on phosphorus partitions between $\mathrm{MgO}$-saturated $\mathrm{CaO}-$ $\mathrm{SiO}_{2}-\mathrm{Fe}_{t} \mathrm{O}-\mathrm{Na}_{2} \mathrm{O}(7 \sim 13 \%)$ slags and liquid iron are indicated by the hatched area. The CaO-equivalencies of $\mathrm{Na}_{2} \mathrm{O}$ on the mole fraction basis obtained in the experiment for the phosphorus distribution between $\mathrm{Na}_{2} \mathrm{O}-\mathrm{SiO}_{2}-\mathrm{CaO}$ slags and carbon-saturated liquid iron melts are shown by the open triangles. The CaO-equivalencies of $\mathrm{Na}_{2} \mathrm{O}$ are independent of the $\mathrm{Na}_{2} \mathrm{O}$ content and considered to be 1.4, although the considerable scatter in the data points was observed. It was found in the previous experiment ${ }^{3)}$ for the $\mathrm{MgO}$-saturated slags containing $\mathrm{CaF}_{2} \mathrm{wt}_{0} \mathrm{~s}$, that the $\mathrm{CaO}$-equivalency of $\mathrm{CaF}_{2}$ was about $0.9,{ }^{4}$ ) but in the present experiment of the slags with the high $\mathrm{CaF}_{2}$ content, the $\mathrm{CaO}$-equivalency of $\mathrm{CaF}_{2}$ was 


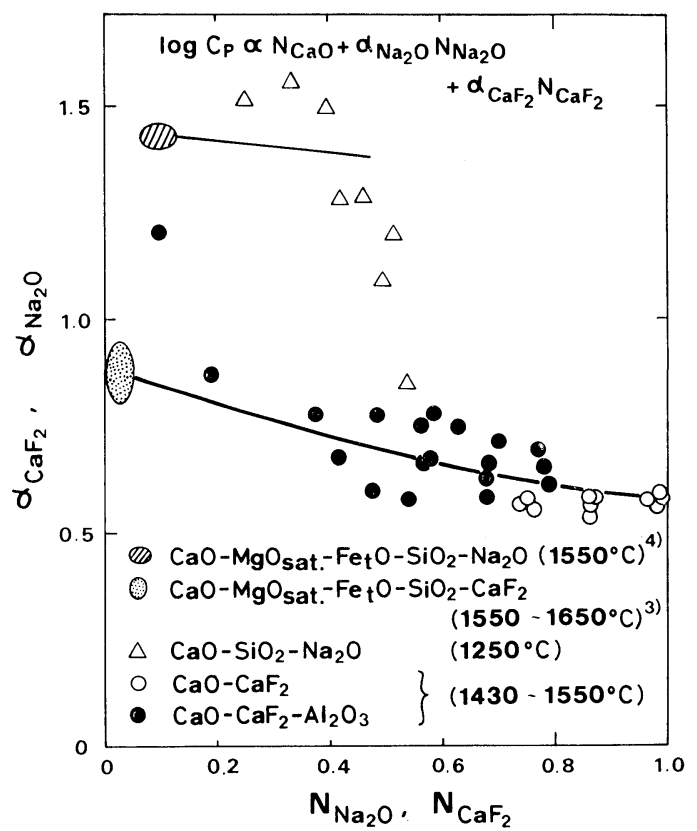

Fig. 8. Effect of $\mathrm{Na}_{2} \mathrm{O}$ or $\mathrm{CaF}_{2}$ on $\log C_{\mathrm{P}}-\mathrm{CaO}$-equivalencies of $\mathrm{Na}_{2} \mathrm{O}$ or $\mathrm{CaF}_{2}$.

found to decrease with an increase in the $\mathrm{CaF}_{2}$ content and found to be 0.6 at the pure $\mathrm{CaF}_{2}$ although with the considerable scatter.

The exchange reactions of fluorides with oxides of difference cations are given as follows:

$$
\begin{gathered}
\mathrm{BaF}_{2}+\mathrm{CaO}=\mathrm{CaF}_{2}+\mathrm{BaO} \\
\Delta G^{\circ}=6140+1.95 T^{15,25)}(\mathrm{cal}) \\
\mathrm{CaF}_{2}+\mathrm{MgO}=\mathrm{MgF}_{2}+\mathrm{CaO} \\
\Delta G^{\circ}=21550-2.50 T^{25,26)}(\mathrm{cal})
\end{gathered}
$$

It can be seen from Figs. 3 and 4 that the values for $\log C_{\mathrm{P}}$ in the $\mathrm{BaF}_{2}-\mathrm{CaO}$ system are smaller than those in the $\mathrm{BaF}_{2}-\mathrm{BaO}$ system and those in the $\mathrm{CaF}_{2}-$ $\mathrm{MgO}$ system are also smaller than those in the $\mathrm{CaF}_{2}-$ $\mathrm{CaO}$ system. The reasons for this can be explained by the facts that the exchange reactions given by Eqs. (13) and (14) do not occur and that the values for $\log C_{\mathrm{P}}$ in the pure oxides decrease in the order of $\mathrm{BaO}>\mathrm{CaO}>\mathrm{MgO}^{4}{ }^{4}$ The fact that the values for $\log C_{\mathrm{P}}$ in the $\mathrm{CaF}_{2}-\mathrm{BaO}$ system are smaller than those in the $\mathrm{CaF}_{2}-\mathrm{CaO}$ system cannot be explained by the difference in the $\log C_{\mathrm{P}}$ for the pure oxides. The experimental points of the slag compositions in the $\mathrm{CaF}_{2}-\mathrm{BaO}$ system were recalculated from Eq. (13) on the assumption that one mole of $\mathrm{CaO}$ was formed from one mole of $\mathrm{BaO}$; that is, about $4 \mathrm{wt} \% \mathrm{CaO}$ was formed from $10 \mathrm{wt} \% \mathrm{BaO}$ on the weight percent basis. The values for $\log C_{\mathrm{P}}$ plotted against the recalculated slag compositions were fo ind to be in good agreement with those obtained in the $\mathrm{CaF}_{2-}$ $\mathrm{CaO}$ system.

Tabuchi and $\mathrm{Sano}^{27)}$ have studied the phosphorus distribution between carbon-saturated $\mathrm{Ag}$ melts and the $\mathrm{CaF}_{2}-\mathrm{CaO}$ slags at $1500{ }^{\circ} \mathrm{C}$ under the $P_{\mathrm{CO}}=$ $0.5 \mathrm{~atm}\left(P_{\mathrm{O}_{2}}=4.56 \times 10^{-17} \mathrm{~atm}\right)$ atmosphere. Their results confirm the exsistence of $\mathrm{P}^{3-}$ ion in addition to $\mathrm{P}^{5+}$ ion, whose content is about one-fourth of $\mathrm{P}^{5+}$ ion.

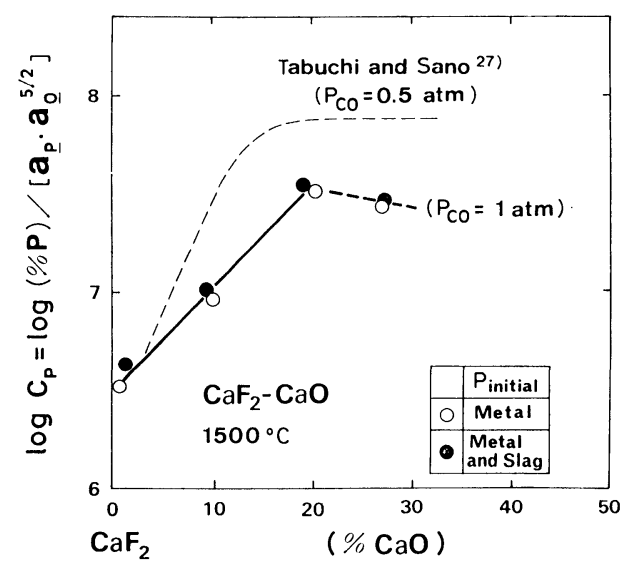

Fig. 9. Comparison with the previous results ${ }^{27}$ ) of the present value for $\log C_{\mathrm{P}}$ in the $\mathrm{CaF}_{2}-\mathrm{CaO}$ system.

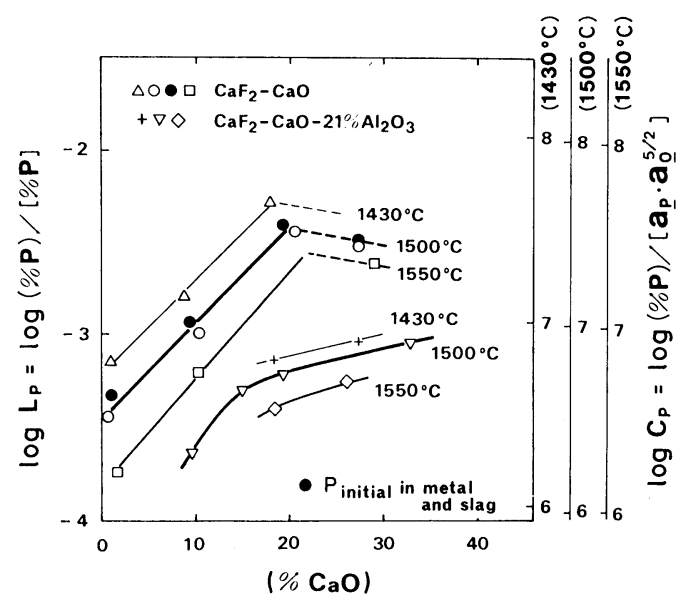

Fig. 10. Temperature dependence of $\log L_{\mathrm{P}}$ in the $\mathrm{CaF}_{2}-$ $\mathrm{CaO}$ and $\mathrm{CaF}_{2}-\mathrm{CaO}-\mathrm{Al}_{2} \mathrm{O}_{3}$ systems.

The values for $\log C_{\mathrm{P}}$ calculated from their phosphate content are also shown in Fig. 9, indicating the close agreement with the present results, although their results for $\log C_{\mathrm{P}}$ are slightly higher than those in this work.

\section{Temperature Dependence of Phosphorus Distribution Ratios}

It is often observed in the practical operation that the phosphorus distribution ratios are dependent on the temperature to a considerable degree. Only a few laboratory data on the temperature dependence of $L_{\mathrm{P}}$ have been reported so far. Figure 10 shows the results for the phosphorus distribution ratios between the $\mathrm{CaF}_{2}-\mathrm{CaO}$ slags and carbon-saturated iron melts at three different temperatures of 1430,1500 and $1550{ }^{\circ} \mathrm{C}$. The results for the $\mathrm{CaF}_{2}-\mathrm{CaO}-\mathrm{Al}_{2} \mathrm{O}_{3}$ slags are also shown in Fig. 10. It is seen from Fig. 10 that the temperature dependence of $L_{\mathrm{P}}$ observed in the $\mathrm{CaF}_{2}-\mathrm{CaO}$ slag is greater than that observed in the $\mathrm{CaF}_{2}-\mathrm{CaO}-\mathrm{Al}_{2} \mathrm{O}_{3}$ slag.

From the results of the phosphorus distribution between $\quad 57 \% \mathrm{Na}_{2} \mathrm{O}-24 \% \mathrm{SiO}_{2}-18 \% \mathrm{PO}_{2.5}-1 \% \mathrm{Fe}_{t} \mathrm{O}$ slags and carbon-saturated iron melts, Tsukihashi et al. ${ }^{22)}$ reported the temperature dependence of the phosphorus distribution ratios as $\log (\% \mathrm{P}) /[\% \mathrm{P}]=$ $21500 / T-11.73\left(1200 \sim 1300{ }^{\circ} \mathrm{C}\right)$. As shown in 


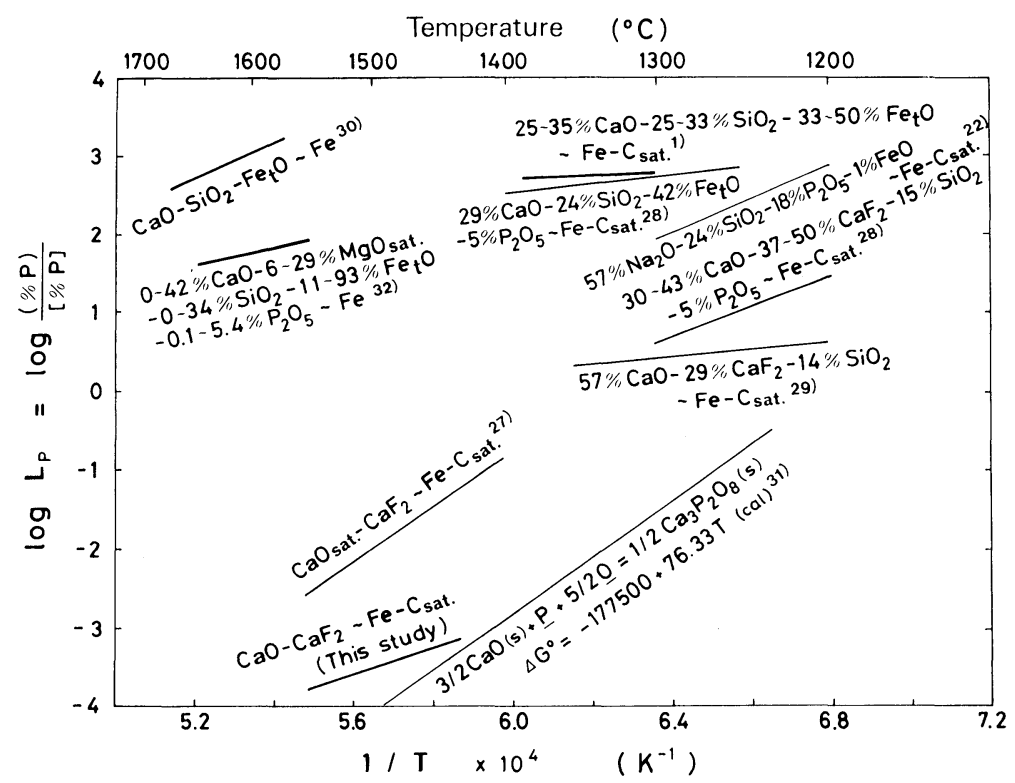

Fig. 11 .

Temperature dependence of $\log L_{\mathrm{P}}$ observed in the fluxes for hot metal pretreatment and liquid steel refining.
Fig. 1, no temperature dependence of $L_{\mathrm{P}}$ was observed in the present $\mathrm{Na}_{2} \mathrm{O}-\mathrm{SiO}_{2}$ slags. The previous results for the temperature dependence of $L_{\mathrm{P}}$ in the $\mathrm{BOF}$ type slags and the slags in a hot metal pretreatment are summarized in Fig. 11, indicating that the temperature dependence of $L_{\mathrm{P}}$ varies with the slag compositions. In order to obtain the accurate results for the variation of $L_{\mathrm{P}}$ with the slag compositions, further works are necessary.

\section{Summary}

The results of the phosphorus distribution between the soda- and lime-based fluxes and carbon-saturated iron melts are summarized as follows;

(1) When $\mathrm{Na}_{2} \mathrm{O}$ is replaced by $\mathrm{MgO}, \mathrm{CaO}$ or $\mathrm{BaO}$ at a given $\mathrm{SiO}_{2}$ content in the $\mathrm{Na}_{2} \mathrm{O}-\mathrm{SiO}_{2}-\mathrm{MO}$ $(\mathrm{M}=\mathrm{Mg}, \mathrm{Ca}, \mathrm{Ba})$ ternary system, the phosphorus distribution ratios were found to decrease. The ratios increase with the addition of $\mathrm{CaO}$ to the $3 \mathrm{Na}_{2} \mathrm{O} \cdot 2 \mathrm{SiO}_{2}$ composition, while they decrease with the addition of $\mathrm{CaF}_{2}$.

(2) The phosphorus distribution ratios in the $\mathrm{MF}_{2}(\mathrm{MF})-\mathrm{MO}\left(\mathrm{M}_{2} \mathrm{O}\right)(\mathrm{M}=\mathrm{Na}, \mathrm{Ba}, \mathrm{Ca}, \mathrm{Mg})$ system decrease in the order of $\mathrm{Na}>\mathrm{Ba}>\mathrm{Ca}>\mathrm{Mg}$.

(3) In the $\mathrm{Na}_{2} \mathrm{O}-\mathrm{SiO}_{2}$ system the phosphorus distribution ratios at 1250 and $1350{ }^{\circ} \mathrm{C}$ were nearly equal to each other, but in the $\mathrm{CaF}_{2}-\mathrm{CaO}\left(-\mathrm{Al}_{2} \mathrm{O}_{3}\right)$ system, they decrease with an increase in the temperature from 1430 to $1550^{\circ} \mathrm{C}$. Their temperature dependence in the $\mathrm{CaF}_{2}-\mathrm{CaO}$ system was found to be greater than those in the $\mathrm{CaF}_{2}-\mathrm{CaO}-\mathrm{Al}_{2} \mathrm{O}_{3}$ system.

\section{REFERENCES}

1) K. Iwasaki, N. Sano and Y. Matsushita: Tetsu-to-Hagané, 67 (1981), 536.

2) H. Suito, R. Inoue and M. Takada: Trans. ISIJ, 21 (1981), 250.

3) H. Suito and R. Inoue: Trans. ISIJ, 22 (1982), 869.

4) H. Suito and R. Inoue: Trans. ISIJ, 24 (1984), 47.

5) H. Suito and R. Inoue: Proc. The 2nd Japan-China Symposium on Science and Technology of Iron and Steel, ISIJ, Tokyo, (1983), 249.
6) R. Inoue and H. Suito: Trans. ISIJ, 22 (1982), 514

7) H. Suito and R. Inoue: Unpublished work.

8) G.J.W. Kor and F. D. Richardson: Trans. AIME, 245 (1969), 319.

9) Y. Shiraishi: Bull. Res. Inst. Mineral Dressing Metal., Tohoku Uni., 31 (1975), 47.

10) K. Nakano and Y. Yoshino: Shin Jikken Kagaku Koza, Maruzen, Tokyo, 9 (1976).

11) M. Ishibashi and M. Tafuse: Japan Analyst, 8 (1959), 588.

12) G. K. Sigworth and J. F. Elliott: Metal Sci., 8 (1974), 298.

13) H. G. Hadrys, M. G. Frohberg and J. F. Chipman: Met. Trans., 1 (1970), 1867.

14) F. Neumann, H. Shenck and W. Patterson: Giesserei, 23 (1959), 1217

15) O. Kubaschewski, E. L. Evance and C. B. Alcock: Metallurgical Thermochemistry, 4th ed., Pergamon Press, New York, (1967).

16) C. Wagner: Met. Trans., 6B (1975), 405.

17) R. Inoue and H. Suito: Trans. ISIJ, 24 (1984), 816.

18) P. N. Smith and M. W. Davies: Trans. Inst. Min. Met., 80 (1971), C87.

19) J. Kramss: Thermodynamische Untersuchungen im System Kupfer-Sauerstoff-Phosphor bei $1200^{\circ} \mathrm{C}$, Doctoral Thesis submitted to Berlin Technical University, (1971).

20) K. Ito and N. Sano: Tetsu-to-Hagané, 69 (1983), 1838.

21) N. Sano: Tetsu-to-Hagané, 69 (1983), 378.

22) F. Tsukihashi, F. Matsumoto and N. Sano: Tetsu-toHagané, 69 (1983), S175; Trans. ISIJ, 23 (1983), B243.

23) E. M. Levin, C. R. Robbins and H. F. McMurdie: Phase Diagrams for Ceramists, Amer. Ceram. Soc., Columbus (1969).

24) A. Bronson and G. R. St. Pierre: Met. Trans., 10B (1979), 375.

25) E. T. Turkdogan: Physical Chemistry of High Temperature Technology, Academic Press, New York, (1980).

26) I. Barin and O. Knacke: Thermochemical Properties of Inorganic Substances, Springer Verlag, Berlin, (1973).

27) S. Tabuchi and N. Sano: Tetsu-to-Hagané, 69 (1983), S280; Trans. ISIJ, 23 (1983), B290.

28) K. Ito and N. Sano: Tetsu-to-Hagané, 69 (1983), 1747.

29) M. Muraki, N. Sano and H. Fukushima: Tetsu-to-Hagané, 70 (1984), S203.

30) G. W. Healy: JISI, 208 (1970), 664.

31) J. B. Bookey: JISI, 172 (1952), 61.

32) H. Suito and R. Inoue: Trans. ISIJ, 24 (1984), 40 